\title{
Numerical Verification Method for Infinite Dimensional Eigenvalue Problems
}

\author{
Kaori NAGATOU \\ Faculty of Mathematics, Kyushu University and \\ PRESTO, Japan Science and Technology Agency \\ E-mail: nagatou@math.kyushu-u.ac.jp
}

Received April 23, 2008

Revised September 20, 2008

\begin{abstract}
We consider an eigenvalue problem for differential operators, and show how guaranteed bounds for eigenvalues (together with eigenvectors) are obtained and how non-existence of eigenvalues in a concrete region can be assured. Some examples for several types of operators will be presented.
\end{abstract}

Key words: numerical verification method, infinite dimensional eigenvalue problem, error estimates

\section{Introduction}

Up to now we have developed a method to enclose and exclude eigenvalues for differential operators $[8,9,10,11,12,13]$. This method is based on Nakao's theory which is known as a numerical verification method for partial differential equations $[14,15,16,17]$, and it has a merit that it could be applied even in case the operator is not self-adjoint. A remarkable point of this eigenvalue enclosing/excluding is to assure an existence and non-existence range of eigenvalues with mathematically rigorous sense. This means not only a reliability of computed eigenpairs but also that such evaluation of eigenvalues (and eigenvectors) can be applied to related other problems, e.g., other numerical verification methods for nonlinear problems or stability analysis of bifurcation phenomenon in hydrodynamics.

This paper aims to show how eigenvalues (and eigenvectors) are enclosed or excluded in mathematically rigorous sense. At first in Section 2, we introduce some eigenvalue enclosure methods, especially for symmetric operators. Our original method is described in Section 3 together with some applications to algebra, other numerical verification methods for nonlinear problems and stability analysis of bifurcation phenomenon in hydrodynamics.

\section{Enclosure methods for symmetric operators}

Finding an eigenvalue and eigenvector of infinite dimensional operator (i.e., an operator defined in an infinite dimensional space) is called as infinite dimensional eigenvalue problem.

Let $H$ be an infinite dimensional Hilbert space with an inner product $\langle\cdot, \cdot\rangle$. For a linear symmetric operator $L: \mathcal{D}(L) \rightarrow H$, we consider the eigenvalue problem

$$
L u=\lambda u, \quad u \in \mathcal{D}(L) \backslash\{0\} .
$$


An eigenvalue of an operator takes an important role to understand a nonlinear phenomenon in science and engineering. Especially, it often becomes a key value when we consider a behavior of dynamical systems.

Several methods to enclose eigenvalues for symmetric operators have been proposed, and now we introduce some of those methods below. Here we assume that there exists an orthonormal basis of $H$ consisting of eigenfunctions of $L$, and that all eigenvalues are bounded below and ordered as $\lambda_{1} \leq \lambda_{2} \leq \cdots$.

\section{Krylov-Weinstein's bounds [4]}

As one of the simplest way of eigenvalue enclosure, Krylov-Weinstein's bounds is well known.

Let $(\tilde{u}, \tilde{\lambda}) \in \mathcal{D}(L) \times \mathbf{R}$ be an approximate eigenpair and compute

$$
\delta \equiv \frac{\|L \tilde{u}-\tilde{\lambda} \tilde{u}\|}{\|\tilde{u}\|} .
$$

Then the interval

$$
[\tilde{\lambda}-\delta, \tilde{\lambda}+\delta]
$$

contains at least one eigenvalue of $L$.

This bound is easy to compute, but the width of the enclosed interval is not so narrow. And it also has another defect that no information is obtained concerning the index of eigenvalue.

\section{Kato-Temple's bounds [6]}

As an improved version of Krylov-Weinstein's bounds, there is a KatoTemple's bounds which was proposed in 1949 [6].

Let $(\tilde{u}, \tilde{\lambda})$ be an approximate eigenpair satisfying

$$
\tilde{\lambda}=\langle L \tilde{u}, \tilde{u}\rangle /\langle\tilde{u}, \tilde{u}\rangle
$$

and compute

$$
\delta \equiv \frac{\|L \tilde{u}-\tilde{\lambda} \tilde{u}\|}{\|\tilde{u}\|} .
$$

For the $n$-th eigenvalue $\lambda_{n}$ with finite multiplicity, suppose that an open interval $(\alpha, \beta)$ does not contain any spectrum except for $\lambda_{n}$. Then for $\rho \in \mathbf{R}$ satisfying $\alpha<\rho<\beta$, we have

$$
\lambda_{n} \in\left[\rho-\frac{\delta^{2}}{\beta-\rho}, \rho+\frac{\delta^{2}}{\rho-\alpha}\right] .
$$

The quality of this bounds is better than Krylov-Weinstein's bounds. Indeed it has an $O\left(\delta^{2}\right)$ quality compared with an $O(\delta)$ quality of Krylov-Weinstein's bounds. But it also has a difficulty that it needs a precise information on eigenvalue distribution in advance, i.e., a (rough) upper bound for $\lambda_{n-1}$ and (rough) lower bound for $\lambda_{n+1}$ are needed to obtain the result. 


\section{Rayleigh-Ritz bounds [4]}

The Rayleigh-Ritz method is well known as a method to obtain very accurate upper bounds for the first $N$ eigenvalues of $L$.

Let $\tilde{u}_{1}, \ldots, \tilde{u}_{N} \in \mathcal{D}(L)$ be linearly independent functions and define two $N \times N$-matrices

$$
\begin{aligned}
& A_{1} \equiv\left(\left\langle L \tilde{u}_{i}, \tilde{u}_{j}\right\rangle\right)_{i, j=1, \ldots, N}, \\
& A_{2} \equiv\left(\left\langle\tilde{u}_{i}, \tilde{u}_{j}\right\rangle\right)_{i, j=1, \ldots, N} .
\end{aligned}
$$

And let $\Lambda_{1} \leq \cdots \leq \Lambda_{N}$ be the eigenvalues of the matrix eigenvalue problem

$$
A_{1} x=\Lambda A_{2} x, \quad x \in \mathbf{R}^{N} \backslash\{0\} .
$$

Then we have

$$
\lambda_{i} \leq \Lambda_{i} \quad(i=1, \ldots, N) .
$$

Being different from Kato-Temple's bounds, this method does not need any a priori information concerning eigenvalue distribution, but it does not give any lower bounds.

\section{Lehmann's bounds [3]}

Concerning the lower bounds for eigenvalues, there is a Lehmann's method as follows.

Let $\tilde{u}_{1}, \ldots, \tilde{u}_{N} \in \mathcal{D}(L)$ be linearly independent functions and suppose that $\Lambda_{N}<\nu \leq \lambda_{N+1}$ holds for a real number $\nu$, where $\Lambda_{N}$ denotes the Rayleigh-Ritz bound. Moreover define three $N \times N$-matrices

$$
\begin{aligned}
& A_{3} \equiv\left(\left\langle L \tilde{u}_{i}, L \tilde{u}_{j}\right\rangle\right)_{i, j=1, \ldots, N} \\
& B_{1} \equiv A_{1}-\nu A_{2} \\
& B_{2} \equiv A_{3}-2 \nu A_{1}+\nu^{2} A_{2}
\end{aligned}
$$

where $A_{1}$ and $A_{2}$ are the same matrices in Rayleigh-Ritz method. If the eigenvalues $\mu_{1} \leq \cdots \leq \mu_{N}$ of the matrix eigenvalue problem

$$
B_{1} x=\mu B_{2} x, \quad x \in \mathbf{R}^{N} \backslash\{0\}
$$

are negative, we have

$$
\lambda_{N+1-i} \geq \nu+\frac{1}{\mu_{i}} \quad(i=1, \ldots, N) .
$$

This lower bound is also sharp, but it also has the same difficulty as KatoTemple's method, i.e., it needs a priori information on the exact eigenvalue $\lambda_{N+1}$. 


\section{Homotopy method [18]}

In order to overcome the difficulty to obtain a priori information on exact eigenvalues, the homotopy method was proposed by Plum in 1990 [18]. In his method a base problem is considered which is related to the given problem, i.e., for the eigenvalue problem for $L$. Here the base problem is chosen so that the eigenvalue distribution of it is already obtained. Let $L_{0}$ be an operator which corresponds to this base problem, then consider a homotopy which connects two operators $L$ and $L_{0}$ :

$$
L_{s} \equiv(1-s) L_{0}+s L, \quad s \in[0,1] .
$$

Then starting from $s=0$ and making use of the continuity and monotonicity of eigenvalues on the parameter $s$, some eigenvalues for $L_{s}$ are enclosed in each step. Finally the first several eigenvalues of $L$ are enclosed when the parameter $s$ reached 1.

Besides these methods, there is an intermediate methods [1, 2], but all these methods are restricted to symmetric operators and cannot be applied to nonsymmetric operators. Moreover, these methods give direct enclosures only for eigenvalues, while eigenfunction enclosures require a bit of a posteriori work. In [20], a general enclosure theory for eigenvalue problems $A u=\lambda B u$ with a closed operator $A$ and a bounded operator $B$, i.e., including non-symmetric problems, was proposed. In the next section, we introduce our method which could be also applied to non-symmetric operators and also provides the eigenvector enclosures directly. (See also [23].)

\section{Enclosure method based on Nakao's theory}

We have developed a method to enclose eigenvalues and eigenvectors for differential operators $[8,9,10]$, which was based on Nakao's verification methods for nonlinear differential equations $[14,15,16,17]$. Our method is also applicable to non-symmetric operators. So far we have applied our enclosure method to enclose eigenpair of symmetric operators and to enclose real eigenvalues and corresponding eigenvectors of a non-symmetric operator.

Now we describe the principle of our method, and show some applications.

\subsection{Eigenvalue enclosing and excluding $\operatorname{method}([8,9,10])$}

Though the following arguments are almost the same as [10], in order to keep this paper to be self-contained, we will give the detailed description below.

We consider a self-adjoint eigenvalue problem:

$$
\begin{cases}-\Delta u+q u=\lambda u & \text { in } \Omega, \\ u=0 & \text { on } \partial \Omega .\end{cases}
$$

Here $\Omega$ is a bounded convex domain in $\mathbf{R}^{2}$ and let $q \in L^{\infty}(\Omega)$. We apply Nakao's method which is known as a numerical verification method for nonlinear problems. 
In what follows, for some integer $m$, let $H^{m}(\Omega)$ denote the $L^{2}$-Sobolev space of order $m$ on $\Omega$. Then, define $H_{0}^{1}(\Omega) \equiv\left\{v \in H^{1}(\Omega) \mid v=0\right.$ on $\left.\partial \Omega\right\}$ with the inner product $\langle u, v\rangle_{H_{0}^{1}} \equiv(\nabla u, \nabla v)_{L^{2}}$ for $u, v \in H_{0}^{1}(\Omega)$, and the norm $\|u\|_{H_{0}^{1}} \equiv\|\nabla u\|_{L^{2}}$ for $u \in H_{0}^{1}(\Omega)$, where $(\cdot, \cdot)_{L^{2}}$ and $\|\cdot\|_{L^{2}}$ represent the inner product and the norm on $L^{2}(\Omega)$, respectively.

Now, let $S_{h}$ be a finite dimensional subspace of $H_{0}^{1}(\Omega)$ dependent on $h$ $(0<h<1)$. Usually, $S_{h}$ is taken to be a finite element subspace with mesh size $h$. Also, let

$$
P_{h 0}: H_{0}^{1}(\Omega) \rightarrow S_{h}
$$

denote the $H_{0}^{1}$-projection defined by

$$
\left(\nabla\left(u-P_{h 0} u\right), \nabla v_{h}\right)_{L^{2}}=0 \text { for all } v_{h} \in S_{h} .
$$

We now assume the following approximation property in $S_{h}$ :

Assumption 1. For any $u \in H^{2}(\Omega) \cap H_{0}^{1}(\Omega)$,

$$
\inf _{\chi \in S_{h}}\|u-\chi\|_{H_{0}^{1}} \leq C_{1} h|u|_{H^{2}}
$$

where

$$
|u|_{H^{2}}^{2} \equiv \sum_{i, j=1}^{2}\left\|\frac{\partial^{2} u}{\partial x_{i} \partial x_{j}}\right\|_{L^{2}}^{2} .
$$

Here, $C_{1}$ is a positive, numerically verified constant which is independent of $h$.

The following lemma is well known [5]:

Lemma 1. For any $\psi$ in $L^{2}(\Omega)$, there exists a unique solution $\phi \in H^{2}(\Omega) \cap$ $H_{0}^{1}(\Omega)$ of the following Poisson equation:

$$
\begin{cases}-\Delta \phi=\psi & \text { in } \Omega, \\ \phi=0 & \text { on } \partial \Omega .\end{cases}
$$

Furthermore, there exists a positive constant $C_{2}$ satisfying

$$
|\phi|_{H^{2}} \leq C_{2}\|\psi\|_{L^{2}}
$$

In particular, if $\Omega$ is a convex polygonal domain, we can set $C_{2}=1$ ([5]).

Since we want to verify the eigenpairs of this problem, we consider the space $H_{0}^{1}(\Omega) \times \mathbf{R}$, and define the innner product $\langle\cdot, \cdot\rangle_{H_{0}^{1} \times \mathbf{R}}$ and the norm $\|\cdot\|_{H_{0}^{1} \times \mathbf{R}}$ by

$$
\begin{aligned}
& \left\langle w_{1}, w_{2}\right\rangle_{H_{0}^{1} \times \mathbf{R}} \equiv\left(\nabla u_{1}, \nabla u_{2}\right)_{L^{2}}+\lambda_{1} \lambda_{2} \\
& \|w\|_{H_{0}^{1} \times \mathbf{R}} \equiv\left(\|u\|_{H_{0}^{1}}^{2}+|\lambda|^{2}\right)^{\frac{1}{2}}
\end{aligned}
$$


respectively, where $w_{i}=\left(u_{i}, \lambda_{i}\right) \in H_{0}^{1}(\Omega) \times \mathbf{R}(i=1,2)$ and $w=(u, \lambda) \in H_{0}^{1}(\Omega) \times \mathbf{R}$. Moreover, let $I_{0}$ and $I$ be the identity map on $H_{0}^{1}(\Omega)$ and $H_{0}^{1}(\Omega) \times \mathbf{R}$, respectively.

We first normalize the problem (3.1) as

$$
\text { find }(\hat{u}, \lambda) \in H_{0}^{1}(\Omega) \times \mathbf{R} \text { s.t. }\left\{\begin{array}{l}
-\Delta \hat{u}+(q-\lambda) \hat{u}=0, \\
\int_{\Omega} \hat{u}^{2} d x=1 .
\end{array}\right.
$$

We define the projection

$$
P_{h}: H_{0}^{1}(\Omega) \times \mathbf{R} \rightarrow S_{h} \times \mathbf{R}
$$

by

$$
P_{h}(u, \lambda) \equiv\left(P_{h 0} u, \lambda\right) .
$$

Now, let $\hat{w}_{h}=\left(\hat{u}_{h}, \hat{\lambda}_{h}\right) \in S_{h} \times \mathbf{R}$ be a finite element solution of (3.5), that is,

$$
\left\{\begin{array}{l}
\left(\nabla \hat{u}_{h}, \nabla \phi_{h}\right)_{L^{2}}=\left(\left(\hat{\lambda}_{h}-q\right) \hat{u}_{h}, \phi_{h}\right)_{L^{2}} \quad \forall \phi_{h} \in S_{h}, \\
\int_{\Omega} \hat{u}_{h}^{2} d x=1 .
\end{array}\right.
$$

We will verify the existence of an eigenvalue and an eigenfunction for (3.5) in the neighborhood of $\left(\bar{u}, \hat{\lambda}_{h}\right)$ satisfying

$$
\begin{cases}-\Delta \bar{u}+\left(q-\hat{\lambda}_{h}\right) \hat{u}_{h}=0 & \text { in } \Omega, \\ \bar{u}=0 & \text { on } \partial \Omega .\end{cases}
$$

Notice that $\bar{u} \in H^{2}(\Omega) \cap H_{0}^{1}(\Omega)$, and $\hat{w}_{h}=P_{h}\left(\bar{u}, \hat{\lambda}_{h}\right)$. We have by $(3.5)$ and (3.7)

$$
\left\{\begin{array}{l}
-\Delta(\hat{u}-\bar{u})=(\lambda-q) \hat{u}-\left(\hat{\lambda}_{h}-q\right) \hat{u}_{h}, \\
\int_{\Omega} \hat{u}^{2} d x=1 .
\end{array}\right.
$$

Defining $v_{0}=\bar{u}-\hat{u}_{h}$, we then have $v_{0} \in S_{h}^{\perp}$, where $S_{h}^{\perp}$ means the orthogonal complement of $S_{h}$ in $H_{0}^{1}(\Omega)$, and we can write

$$
\bar{u}=\hat{u}_{h}+v_{0}, \quad \text { where } \hat{u}_{h} \in S_{h} \text { and } v_{0} \in S_{h}^{\perp} .
$$

Here we use a posteriori estimates for $v_{0}$ as follows.

Let $S_{h}^{*} \subset H^{1}(\Omega)$ be a finite element subspace whose basis consists of the union of the basis on $S_{h}$ and the base functions having nonzero values on the boundary 
$\partial \Omega$. Define $\bar{\nabla} \hat{u}_{h} \in S_{h}^{*} \times S_{h}^{*}$, a vector function in two dimension, by the $L^{2}$-projection of $\nabla \hat{u}_{h} \in L^{2} \times L^{2}$ to $S_{h}^{*} \times S_{h}^{*}$. Then, define $\bar{\Delta} \hat{u}_{h} \in L^{2}(\Omega)$ by

$$
\bar{\Delta} \hat{u}_{h} \equiv \nabla \cdot \bar{\nabla} \hat{u}_{h}
$$

We then obtain the following estimation (cf. [24]):

$$
\left\|v_{0}\right\|_{H_{0}^{1}} \equiv\left\|\nabla \hat{u}_{h}-\bar{\nabla} \hat{u}_{h}\right\|+C_{0} h\left\|\bar{\Delta} \hat{u}_{h}+\left(\hat{\lambda}_{h}-q\right) \hat{u}_{h}\right\|
$$

where $C_{0} \equiv C_{1} C_{2}$. Using the well-known Aubin-Nitsche trick ([7]), we can estimate the $L^{2}$ norm of $v_{0}$ as

$$
\left\|v_{0}\right\|_{L^{2}} \leq C_{0} h\left\|v_{0}\right\|_{H_{0}^{1}}
$$

Now, in order to verify solutions $(\hat{u}, \lambda)$ of $(3.5)$ near $\left(\bar{u}, \hat{\lambda}_{h}\right)$, representing

$$
\hat{u}=\bar{u}+\tilde{u}, \quad \lambda=\hat{\lambda}_{h}+\tilde{\lambda}
$$

we can rewrite (3.8) as

$$
\begin{aligned}
& -\Delta \tilde{u}=\left(\hat{\lambda}_{h}+\tilde{\lambda}-q\right)\left(\tilde{u}+\hat{u}_{h}+v_{0}\right)-\left(\hat{\lambda}_{h}-q\right) \hat{u}_{h}, \\
& \int_{\Omega}\left(\tilde{u}+\hat{u}_{h}+v_{0}\right)^{2} d x=1 .
\end{aligned}
$$

Thus using the following compact map on $H_{0}^{1}(\Omega) \times \mathbf{R}$

$$
\begin{aligned}
& F(\tilde{u}, \tilde{\lambda}) \\
& \equiv\left((-\Delta)^{-1}\left\{\left(\hat{\lambda}_{h}+\tilde{\lambda}-q\right)\left(\tilde{u}+\hat{u}_{h}+v_{0}\right)-\left(\hat{\lambda}_{h}-q\right) \hat{u}_{h}\right\}, \tilde{\lambda}+\int_{\Omega}\left(\tilde{u}+\hat{u}_{h}+v_{0}\right)^{2} d x-1\right),
\end{aligned}
$$

where $(-\Delta)^{-1}$ means the solution operator for Poisson equation with homogeneous boundary condition, we have the fixed point equation for $w=(\tilde{u}, \tilde{\lambda})$

$$
w=F(w) .
$$

We now make the following assumption.

Assumption 2. Set $\rho \equiv\left(-v_{0}, 0\right)$ and define $F^{\prime}(\rho)$ as the Fréchet derivative of $F$ at $\rho$. Assume that restriction to $S_{h} \times \mathbf{R}$ of the operator $P_{h}\left[I-F^{\prime}(\rho)\right]: H_{0}^{1}(\Omega) \times$ $\mathbf{R} \rightarrow S_{h} \times \mathbf{R}$ has an inverse

$$
\left[I-F^{\prime}(\rho)\right]_{h}^{-1}: S_{h} \times \mathbf{R} \rightarrow S_{h} \times \mathbf{R} .
$$


This assumption can be numerically checked in the actual computation.

Now we decompose (3.10) into the finite and the infinite dimensional parts:

$$
\left\{\begin{array}{l}
P_{h} w=P_{h} F(w) \\
\left(I-P_{h}\right) w=\left(I-P_{h}\right) F(w) .
\end{array}\right.
$$

And we use the Newton-like method only for the former part of (3.11), that is, we define the Newton-like operator

$$
N_{h}(w) \equiv P_{h} w-\left[I-F^{\prime}(\rho)\right]_{h}^{-1}\left(P_{h} w-P_{h} F(w)\right) .
$$

We next define the operator

$$
T: H_{0}^{1}(\Omega) \times \mathbf{R} \rightarrow H_{0}^{1}(\Omega) \times \mathbf{R}
$$

as

$$
T(w) \equiv N_{h}(w)+\left(I-P_{h}\right) F(w) .
$$

Then $T$ becomes a compact map on $H_{0}^{1}(\Omega) \times \mathbf{R}$, and

$$
w=T(w) \Longleftrightarrow w=F(w)
$$

holds.

An arbitrary element $w \in H_{0}^{1}(\Omega) \times \mathbf{R}$ can be uniquely written as

$$
w=\left(v_{h}, \mu\right)+\left(v_{\perp}, 0\right), \quad\left(v_{h}, \mu\right) \in S_{h} \times \mathbf{R}, \quad\left(v_{\perp}, 0\right) \in S_{h}^{\perp} \times\{0\}
$$

with

$$
v_{h}=\sum_{j=1}^{M} v_{j} \phi_{j}, \quad\left\{\phi_{1}, \ldots, \phi_{M}\right\}: \text { basis of } S_{h} .
$$

And for $w$ in (3.14) we use the following notation:

$$
\begin{aligned}
& (w)_{i} \equiv\left|v_{i}\right|, \quad i=1, \ldots, M, \\
& (w)_{M+1} \equiv\left\|v_{\perp}\right\|_{H_{0}^{1}}, \\
& (w)_{M+2} \equiv|\mu| .
\end{aligned}
$$

Now, we intend to find a solution to (3.5) in a set $W$, referred to as a "candidate set." Taking a vector $\left(W_{1}, \ldots, W_{M+2}\right)^{\mathrm{t}}$ such that $W_{i}>0(i=1, \ldots, M+2)$, a candidate set $W$ is defined by

$$
W \equiv\left\{w \in H_{0}^{1}(\Omega) \times \mathbf{R} \mid(w)_{i} \leq W_{i}(i=1, \ldots, M+2)\right\}
$$


Now let $T^{\prime}$ be the Fréchet derivative of $T$. Then we choose two vectors

$$
\left(Y_{1}, \ldots, Y_{M+2}\right)^{\mathrm{t}}, \quad Y_{i}>0(i=1, \ldots, M+2)
$$

and

$$
\left(Z_{1}, \ldots, Z_{M+2}\right)^{\mathrm{t}}, \quad Z_{i}>0(i=1, \ldots, M+2)
$$

such that

$$
\begin{array}{ll}
(T(0))_{i} \leq Y_{i}, & i=1, \ldots, M+2, \\
\left(T^{\prime}\left(w_{1}\right) w_{2}\right)_{i} \leq Z_{i}, & i=1, \ldots, M+2, \forall w_{1}, w_{2} \in W .
\end{array}
$$

The verification condition is described in the following theorem.

THEOREM 1. If a candidate set $W$, defined by (3.15), satisfies

$$
Y_{i}+Z_{i}<W_{i} \quad(i=1, \ldots, M+2),
$$

then there exists a fixed point of $T$ in

$$
K \equiv\left\{v \in H_{0}^{1}(\Omega) \times \mathbf{R} \mid(v)_{i} \leq Y_{i}+Z_{i}(i=1, \ldots, M+2)\right\} .
$$

Moreover, this fixed point is unique within the set $W$.

By this method we can uniquely enclose an eigenpair $(\hat{u}, \lambda)$ in the set $W$. The author further extended this method by using an infinite dimensional homotopy method, and obtained the local uniqueness of eigenvalue and eigenvector respectively as follows:

THEOREM 2. If a set $W=U \times \Lambda \subset H_{0}^{1}(\Omega) \times \mathbf{R}$ satisfies the conditions in Theorem 1, then we have

i) $\exists 1 u^{*}:$ eigenfunction s.t. $u^{*}-\bar{u} \in U, \int_{\Omega}\left(u^{*}\right)^{2} d x=1$,

ii) $\exists 1 \lambda^{*}$ : eigenvalue s.t. $\lambda^{*}-\hat{\lambda}_{h} \in \Lambda$,

iii) $\quad F\left(u^{*}-\bar{u}, \lambda^{*}-\hat{\lambda}_{h}\right)=\left(u^{*}-\bar{u}, \lambda^{*}-\hat{\lambda}_{h}\right)$,

iv) $\lambda^{*}$ : geometric simple eigenvalue.

(See [10] for the proof of Theorem 1 and Theorem 2.)

It is remarkable that this extended method can assure that the enclosed eigenvalue is simple in mathematically rigorous sense.

Moreover we have proposed a method to exclude an eigenvalue in a concrete interval, i.e., to prove that there is no eigenvalue in such an interval. This could be done as follows.

Let $\Lambda$ be a narrow interval in which we want to exclude any eigenvalues. Then consider the linear equation

$$
\begin{cases}-\Delta u+q u=\Lambda u & \text { in } \Omega, \\ u=0 & \text { on } \partial \Omega .\end{cases}
$$


Since the equation (3.18) has a trivial solution $u \equiv 0$, if we could prove the uniqueness of the solution of (3.18) then the non-existence of eigenvalues in $\Lambda$ would be confirmed.

Now, we describe the manner how to validate the uniqueness of the solutions for (3.18). We consider the following second-order elliptic boundary value problem for a fixed $\lambda \in \Lambda$ :

$$
\begin{cases}-\Delta u=(\lambda-q) u & \text { in } \Omega, \\ u=0 & \text { on } \partial \Omega .\end{cases}
$$

Using the following compact map on $H_{0}^{1}(\Omega)$

$$
F(\lambda) u \equiv(-\Delta)^{-1}(\lambda-q) u,
$$

we can rewrite (3.19) as follows:

$$
F(\lambda) u=u
$$

In the same way as before, we set

$$
\begin{aligned}
& N_{h 0}(\lambda) u \equiv P_{h 0} u-[I-F(\lambda)]_{h 0}^{-1}\left(P_{h 0} u-P_{h 0} F(\lambda) u\right), \\
& T(\lambda) u \equiv N_{h 0}(\lambda) u+\left(I-P_{h 0}\right) F(\lambda) u,
\end{aligned}
$$

where we supposed that restriction to $S_{h}$ of the operator $P_{h 0}[I-F(\lambda)]: H_{0}^{1}(\Omega) \rightarrow$ $S_{h}$ has an inverse $[I-F(\lambda)]_{h 0}^{-1}$, and this can be checked in the actual computation. Then $T(\lambda)$ is a compact linear map on $H_{0}^{1}(\Omega)$ and following equivalence relation holds:

$$
T(\lambda) u=u \Longleftrightarrow F(\lambda) u=u .
$$

We have the following theorem:

TheOREm 3. If there exists a non-empty, closed, bounded and convex set $U \subset H_{0}^{1}(\Omega)$ satisfying $T(\lambda) U \stackrel{\circ}{\subset} U$, then there exists a unique solution $u \in H_{0}^{1}(\Omega)$ of $F(\lambda) u=u$.

Here, $M_{1} \stackrel{\circ}{\subset} M_{2}$ implies $\bar{M}_{1} \subset \stackrel{\circ}{M_{2}}$ for any sets $M_{1}, M_{2}$.

Proof. Consider $v$ satisfying $T(\lambda) v=v$. Since $T(\lambda)$ is a linear operator, for any $c \in \mathbf{R}$ we have

$$
T(\lambda)(c v)=c T(\lambda) v=c v .
$$

If $v \neq 0$, we can choose $\hat{c} \in \mathbf{R}$ satisfying

$$
\hat{c} v \in \partial U \text {. }
$$

But this contradicts with $T(\lambda) U \stackrel{\circ}{\subset} U$ and (3.22). Therefore $v=0$. That is, $u=0$ is a unique solution of $F(\lambda) u=u$. 
By Theorem 3, if there exists a closed, bounded and convex set $U \subset H_{0}^{1}(\Omega)$

satisfying $T(\lambda) U \stackrel{\circ}{\subset} U$ for each $\lambda \in \Lambda$, then it means that we validated the uniqueness for the trivial solution $u=0$ of (3.19). We use an interval arithmetic to treat all $\lambda \in \Lambda$ in a computer. Verification procedure in a computer is same as above.

Although we cannot take so wide interval as $\Lambda$ (usually $10^{-1}-10^{-3}$, depending on the problem), by changing $\Lambda$ little by little we can cover a rather wide range which we want to prove the non-existence of eigenvalues. These enclosing and excluding methods will be able to apply to the problem in $\mathbf{R}^{3}$.

\subsection{Applications}

Now we present some examples to which we have applied our method so far.

\section{Application 1: A numerical verification of solutions for nonlinear elliptic problems [9]}

We consider the nonlinear elliptic boudary value problem:

$$
\begin{cases}-\Delta u=f(u) & \text { in } \Omega, \\ u=0 & \text { on } \partial \Omega,\end{cases}
$$

where $\Omega$ is a bounded convex domain in $\mathbf{R}^{2}$ and $f: H_{0}^{1}(\Omega) \rightarrow L^{2}(\Omega)$ satisfies some suitable conditions (cf. [9]). In [9] we evaluated the norm of the inverse operator of the linearized operator by making use of our eigenvalue excluding method, and used the infinite dimensional Newton's method which is based on Plum's method [19]. This can be regarded as a combined method between Nakao's method and Plum's method.

\section{Application 2: Linearized eigenvalue problem at an exact solution of nonlinear problems [11]}

We consider the follwing problem:

$$
\text { find }(u, v, \lambda) \in H_{0}^{1}(\Omega) \times H_{0}^{1}(\Omega) \times \mathbf{R} \text { s.t. }\left\{\begin{array}{l}
-\Delta u=f(u), \\
-\Delta v-f^{\prime}(u) v=\lambda v \\
\int_{\Omega} v^{2} d x=1,
\end{array}\right.
$$

where $\Omega$ and $f$ are same as in Application 1.

This type of problem is important to analyze the stability of a solution or bifurcation point itself in mathematically rigorous sense. By enclosing the triple $(u, v, \lambda)$ of $(3.24)$, we can obtain an exact solution of nonlinear equation and eigenpair of the operator which was linearized at the exact solution. 


\section{Application 3: Eigenvalue problem for non-commutative harmonic oscil- lators [12]}

The purpose of this research is to develop a verified numerical method for computing the eigenvalues and eigenfunctions of the following system:

$$
Q_{(\alpha, \beta)} \equiv I_{(\alpha, \beta)}\left(-\frac{1}{2} \frac{d^{2}}{d x^{2}}+\frac{x^{2}}{2}\right)+J\left(x \frac{d}{d x}+\frac{1}{2}\right) .
$$

Here $x \in \mathbf{R}$ and the matrices $I_{(\alpha, \beta)}$ and $J$ are given by

$$
I_{(\alpha, \beta)} \equiv\left(\begin{array}{cc}
\alpha & 0 \\
0 & \beta
\end{array}\right), J \equiv\left(\begin{array}{cc}
0 & -1 \\
1 & 0
\end{array}\right) \in \operatorname{Mat}_{2}(\mathbf{R}),
$$

and $\alpha$ and $\beta$ are positive real constants satisfying $\alpha \beta>1$. It is known that $Q_{(\alpha, \beta)}$ defines a self-adjoint positive definite operator, and has a discrete spectrum. Since the spectrum is defined via non-commuting two matrices $I_{(\alpha, \beta)}$ and $J$ for $\alpha \neq \beta$, the system is called by the non-commutative harmonic oscillator.

In case of $\alpha=\beta$, the eigenvalue is determined as

$$
\lambda_{n}=(n+1 / 2) \sqrt{\alpha^{2}-1} \quad(\lambda \in \mathbf{N})
$$

by representation theory, but it is unknown how to describe explicitly the eigenstate, in case $\alpha \neq \beta$. In [12] the spectral method using Hermite functions was used together with our enclosure method, and we obtained very accurate enclosure results in case that $\alpha$ is different from $\beta$. Moreover we have proved that some enclosed eigenvalues have multiplicity 2. Basically our method cannot be applied to enclose multiple eigenvalues, but in this case we could make use of the parity of eigenfunctions and monotonicity of eigenvalues. We can say that this application became a good example of computer assisted proof in pure mathematics.

\section{Application 4: Kolmogorov problem [13]}

We consider the following Navier-Stokes equations

$$
\left\{\begin{array}{l}
\frac{\partial u}{\partial t}+u \frac{\partial u}{\partial x}+v \frac{\partial u}{\partial y}=\nu \Delta u-\frac{1}{\rho} \frac{\partial p}{\partial x}+\gamma \sin \left(\frac{\pi y}{b}\right) \\
\frac{\partial v}{\partial t}+u \frac{\partial v}{\partial x}+v \frac{\partial v}{\partial y}=\nu \Delta v-\frac{1}{\rho} \frac{\partial p}{\partial y} \\
\frac{\partial u}{\partial x}+\frac{\partial v}{\partial y}=0
\end{array}\right.
$$

where $(u, v), \rho, p$ and $\nu$ are velocity vector, mass density, pressure and kinematic viscosity, respectively and $\gamma$ is a constant representing the strength of the sinusoidal outer force. The flow region is a rectangle $[-a, a] \times[-b, b]$ and the periodic boundary condition is imposed in both directions. We define the aspect ratio $\alpha$ as $b / a$.

We have presented a rigorous theorem which proves the stability of certain solutions by the verified computation. The linearized eigenvalue problem arising 
in this problem is not self-adjoint and, accordingly, it is quite difficult to treat theoretically.

It is known that nontrivial solutions bifurcate from the basic solution $(u, v)=$ $(\sin y, 0)$ at a certain Reynolds number if and only if $0<\alpha<1$. If $\alpha$ is small enough or close to unity, then the stability of the bifurcating solution could be proved mathematically. However, stability in the intermediate range of $\alpha$ is very difficult to prove. We therefore took a new approach to this stability problem by employing the theory of verified computation. Our result shows that the stability is rigorously verified for the cases of $\alpha=0.4,0.7$, and 0.8 . Our method can be applied, in principle, to any $\alpha \in(0,1)$.

In [13] we reformulated above problem using a stream function and enclosed an eigenfunction corresponding to the zero eigenvalue as well as the Reynolds number which attain the eigenvalue "zero." Using the results we proved the stability of a bifurcating solution. This is also a good example of computer assisted proof for the problem which is difficult to treat theoretically.

REMARK. Our enclosure method proposed in $[8,10]$ needs the simplicity of the aiming eigenvalues. (Of course such information is not needed in advance.) It means that in principle multiple eigenvalues cannot be enclosed by this method, while the Rayleigh-Ritz and the Lehmann method have no problem with multiple eigenvalues. Concerning the enclosing multiple eigenvalues, see also [22]. In [22], for the eigenvalue problem $L u=\lambda u$, let $n$ be an expected multiplicity of an eigenvalue $\lambda$ and consider the following system:

$$
L Y=Y M, \quad Y \equiv\left(y_{1}, \ldots, y_{n}\right), \quad M \equiv\left(\begin{array}{ccc}
m_{11} & \cdots & m_{1 n} \\
\vdots & \ddots & \vdots \\
m_{n 1} & \cdots & m_{n n}
\end{array}\right)
$$

for $Y_{i} \in H_{0}^{1}(\Omega)$ and $m_{i j} \in \mathbf{R}$. In their mehod the multiple eigenvalue and the basis of corresponding invariant subspace are verified by enclosing a solution $(Y, M) \in$ $\left(H_{0}^{1}(\Omega)\right)^{n} \times \mathbf{R}^{n^{2}}$. This method is an extension of the enclosing method for multiple eigenvalues of matrix [21].

\section{Conclusion}

We have presented a numerical verification method to enclose and exclude eigenvalues of differential operators. This method is based on Nakao's verification method for nonlinear PDEs, and several examples were shown. By using this method, we could enclose an eigenvalue (together with proving its simplicity) and prove the non-existence of eigenvalue in some concrete region, and these verified eigenpairs could be used in other applications with a mathematically rigorous sense.

Our method was also applied to a problem to exclude eigenvalues in spectral gaps of one dimensional Schrödinger operator. This will be presented in the forthcoming paper soon. 
Acknowledgment. The author would like to express many thanks to referees for their important and useful suggestions to improve this paper. This work was supported by a Grant-in-Aid from the Ministry of Education, Culture, Sports, Science and Technology of Japan (No. 16104001, No. 20224001, No. 18740055).

\section{References}

[1] N.W. Bazley and D.W. Fox, A procedure for estimating eigenvalues. Journal of Mathematical Physics, 3 (1962), 469-471.

[ 2 ] C. Beattie and F. Goerisch, Methods for computing lower bounds to eigenvalues of selfadjoint operators. Numerische Mathematik, 72 (1995), 143-172.

[ 3 ] H. Behnke and F. Goerisch, Inclusions for Eigenvalues of Selfadjoint Problems. Topics in Validated Computations, J. Herzberger (ed.), Studies in Computational Mathematics, Elsevier, Amsterdam, 1994.

[ 4 ] F. Chatelin, Spectral Approximation of Linear Operators. Academic Press, New York, 1983.

[ 5 ] P. Grisvard, Elliptic Problems in Nonsmooth Domains. Pitman Monographs and Survays in Pure and Applied Mathematics, 24, London, 1985.

[6 ] T. Kato, On the upper and lower bounds of eigenvalues, Journal of the Physical Society of Japan, 4 (1949), 334-339.

[ 7 ] M. Kř́́žek and P. Neittaanmäki, Finite Element Approximation of Variational Problems and Applications. Longman Scientific and Technical, Harlow, 1990.

[8] M.T. Nakao, N. Yamamoto and K. Nagatou, Numerical verifications for eigenvalues of second-order elliptic operators. Japan Journal of Industrial and Applied Mathematics, 16 (1999), 307-320.

[9] K. Nagatou, N. Yamamoto and M.T. Nakao, An approach to the numerical verification of solutions for nonlinear elliptic problems with local uniqueness. Numerical Functional Analysis and Optimization, 20 (1999), 543-565.

[10] K. Nagatou, A numerical method to verify the elliptic eigenvalue problems including a uniqueness property. Computing, 63 (1999), 109-130.

[11] K. Nagatou and M.T. Nakao, An enclosure method of eigenvalues for the elliptic operator linearlized at an exact solution of nonlinear problems. A special issue on linear algebra in self-validating methods, Linear Algebra and Its Applications, 324 (2001), 81-106.

[12] K. Nagatou, M.T. Nakao and M. Wakayama, Verified numerical computations for eigenvalues of non-commutative harmonic oscillators. Numerical Functional Analysis and Optimization, 23 (2002), 633-650.

[13] K. Nagatou, A computer-assisted proof on the stability of the Kolmogorov flows of incompressible viscous fluid. Journal of Computational and Applied Mathematics, 169 (2004), 33-44.

[14] M.T. Nakao, A numerical approach to the proof of existence of solutions for elliptic problems. Japan Journal of Applied Mathematics, 5 (1988), 313-332.

[15] M.T. Nakao, A numerical approach to the proof of existence of solutions for elliptic problems II. Japan Journal of Applied Mathematics, 7 (1990), 477-488.

[16] M.T. Nakao and N. Yamamoto, Self-Validating Methods (in Japanese). Nihonhyoron-sha, 1998.

[17] M.T. Nakao, Numerical verification methods for solutions of ordinary and partial differential equations. Numerical Functional Analysis and Optimization, 22 (2001), 321-356.

[18] M. Plum, Eigenvalue inclusions for second-order ordinary differential operators by a numerical homotopy method. Journal of Applied Mathematics and Physics (ZAMP), 41 (1990), 205-226.

[19] M. Plum, Explicit $H_{2}$-estimates and pointwise bounds for solutions of second-order elliptic boundary value problems. Journal of Mathematical Analysis and Applications, 165 (1992), 36-61.

[20] M. Plum and J. Lahmann, A computer-assisted instability proof for the Orr-Sommerfeld equation with Blasius profile. ZAMM, 84 (2004), 188-204.

[21] S.M. Rump, Computational numerical bounds for multiple or nearly multiple eigenvalues. Linear Algebra and Its Applications, 324 (2001), 209-226. 
[22] K. Toyonaga, M.T. Nakao and Y. Watanabe, Verified numerical computations for multiple and nearly multiple eigenvalues of elliptic operators. Journal of Computational and Applied Mathematics, 147 (2002), 175-190.

[23] Y. Watanabe, M. Plum and M.T. Nakao, A computer-assisted instability proof for the Orr-Sommerfeld problem with Poiseuille flow. Preprint.

[24] N. Yamamoto and M.T. Nakao, Numerical verifications for solutions to elliptic equations using residual iterations with a high order finite element. Journal of Computational and Applied Mathematics, 60 (1995), 271-279. 
